\title{
AVALIAÇÃO NUTRICIONAL E RISCO CARDIOVASCULAR EM IDOSOS ATENDIDOS NA CLÍNICA DE NUTRIÇÃO DO UNICESUMAR-CENTRO UNIVERSITÁRIO DE MARINGÁ
}

\author{
Magda Pires Corrêa Rivabene Aquino ${ }^{1}$; Rose Mari Bennemann ${ }^{2}$ \\ 1 Acadêmica do Curso de Nutrição, UNICESUMAR, Maringá-PR. Bolsista do \\ PROBIC-UniCesumar magda.molli@gmail.com \\ 2 Orientadora, Dra, Docente do Mestrado em Promoção da Saúde -UNICESUMAR. \\ Pesquisadora do Instituto Cesumar de Ciência, Tecnologia e Inovação - ICETI.
}

Recebido em: 02/10/2017 - Aprovado em: 21/11/2017 - Publicado em: 05/12/2017 DOI: 10.18677/EnciBio_2017B94

\begin{abstract}
RESUMO
A pesquisa teve como objetivo avaliar as condições nutricionais e o risco cardiovascular em idosos atendidos na clínica de nutrição do UNICESUMAR-Centro Universitário de Maringá/PR. Para tanto, foi realizado um estudo transversal, quantitativo e retrospectivo, com a utilização de dados secundários, com idosos (idade $\geq 60$ anos), de ambos os sexos. Foram consultados os prontuários referentes a primeira consulta dos idosos que buscaram atendimento na clínica de nutrição, no período de fevereiro de 2014 a dezembro de 2015. A avaliação nutricional foi determinada por meio do índice de massa corporal (IMC) e o risco cardiovascular por meio da medida da circunferência da cintura. Foram analisados 34 prontuários. A idade variou entre 60 e 82 anos. As mulheres foram as que mais procuraram atendimento nutricional (22 atendimentos) e que apresentaram maior percentual $(63,64 \%)$ de indivíduos classificados com obesidade. Em relação ao risco cardiovascular, os homens apresentaram percentual maior $(83,33 \%)$ de indivíduos com risco cardiovascular. Concluiu-se que os idosos estão com o estado nutricional inadequado e apresentam risco cardiovascular aumentado. Outros estudos necessitam ser realizados e outros parâmetros analisados, para que se possa determinar com exatidão a saúde desses idosos.
\end{abstract}

PALAVRAS-CHAVE: Circunferência cintura; Envelhecimento; Índice massa corporal.

\section{NUTRITIONAL EVALUATION AND CARDIOVASCULAR RISK IN ELDERLY ATTENDED IN THE NUTRITION CLINIC OF UNICESUMAR-CENTRO UNIVERISTÁRIO DE MARINGÁ}

\footnotetext{
ABSTRACT

The objective of this study was to evaluate nutritional conditions and cardiovascular risk in the elderly attended at the nutrition clinic of UNICESUMAR-Centro Universitário de Maringá / PR. A cross-sectional, quantitative, retrospective study was performed using secondary data, with elderly (age $\geq 60$ years) of both sexes. The records to the first consultation of the elderly who sought care at the nutrition ENCICLOPÉDIA BIOSFERA, Centro Científico Conhecer - Goiânia, v.14 n.26; p.1111 2017
} 
clinic were consulted from February 2014 to December 2015. Nutritional assessment was determined by means of body mass index (BMI) and cardiovascular risk by measuring waist circumference. A total of 34 medical records were analyzed. Age ranged from 60 to 82 years. Women were the ones that sought the most nutritional care (22 visits) and presented the highest percentage (63.64\%) of individuals classified as obese. Regarding cardiovascular risk, men presented a higher percentage $(83.33 \%)$ of individuals with cardiovascular risk. It is concluded that the elderly are inadequate nutritional status and present an increased cardiovascular risk. Other studies need to be performed and other parameters analyzed, so that the health of these elderly people can be accurately determined.

KEYWORDS: Aging; Body mass index; Waist circumference.

\section{INTRODUÇÃO}

A população mundial está envelhecendo, em vista disso, uma nova dinâmica demográfica está surgindo. Em 2050, os idosos, indivíduos com 60 anos e mais, corresponderão a $21 \%$ da população mundial, mostrando um aumento expressivo quando comparados com os 8\% em 1950 e 12\% em 2013. Da mesma forma, podese observar o aumento progressivo dos idosos mais velhos, especialmente os de 80 anos e mais, que proporcionalmente crescem mais rápido do que os outros grupos etários (ILC-Brasil, 2015).

O Brasil, que apresentava uma população jovem, a partir dos anos 60 passou a modificar o perfil demográfico, dando início a um processo contínuo de estreitamento da base da pirâmide etária, revelando o envelhecimento da população. Fatores como: aumento da expectativa de vida seguido de declínio da fecundidade e controle das doenças transmissíveis foram vitais para esse processo (ORTOLANI; GOULART, 2015).

Segundo o relatório de estado global sobre doenças não transmissíveis 2014, da Organização Mundial da Saúde, dos 38 milhões de vidas perdidas em 2012 por doenças crônicas não transmissíveis (DCNT) 16 milhões ou 42\% eram prematuras e evitáveis e que com políticas governamentais orientadas para a restrição do consumo de tabaco, consumo nocivo do álcool, dietas pouco saudáveis e sedentarismo, o número de mortes por DCNT pode ser significativamente reduzido (WHO, 2014).

As principais DCNT são as doenças cardiovasculares, cânceres, diabetes, enfermidades respiratórias crônicas e doenças neuropsiquiátricas. Estas constituem o problema de maior magnitude na saúde pública, sendo responsáveis por um número elevado de mortes antes do 70 anos e perda da qualidade de vida, gerando incapacidades de alto grau de limitação em seus portadores. No Brasil, as DCNT respondem por mais de $70 \%$ das causas de morte (IBGE, 2013a). Em estudos realizados com os países da América Latina, o Brasil foi o que apresentou os maiores índices de mortalidade por Acidente vascular cerebral (AVC), sendo a principal causa de mortes entre as mulheres (GARRITANO 2012).

Segundo o ILC-Brasil (2015), a população mundial está envelhecendo. Por isso, é importante conhecer e compreender os fatores que podem influenciar de forma direta ou indireta a saúde do idoso, para que dessa maneira sejam realizados programas de educação nutricional adequados, atendendo a real necessidade. $O$ objetivo da presente pesquisa foi verificar o estado nutricional e risco cardiovascular de pacientes idosos atendidos na Clínica de Nutrição do UNICESUMAR- Centro Universitário de Maringá. 


\section{MATERIAL E MÉTODOS}

O estudo foi de natureza transversal, quantitativo e retrospectivo, realizado com a utilização de dados secundários. Foram utilizados prontuários referentes ao primeiro atendimento de indivíduos idosos (idade $\geq 60$ anos), de ambos os sexos, atendidos na Clínica de Nutrição do UNICESUMAR- Centro Universitário de Maringá, no período de fevereiro de 2014 a dezembro de 2015.

A pesquisa foi aprovada pelo Comitê de Ética em Pesquisa do Centro Universitário de Maringá - UNICESUMAR, sob o número do parecer consubstanciado 1.745.929. Os dados antropométricos (peso, estatura e circunferência da cintura (CC)) e os demográficos (idade e sexo) foram coletados e anotados em formulário próprio. A faixa etária foi agrupada em: 60-69 anos e $\geq 70$ anos para ambos os sexos. Foram excluídos os prontuários que não atenderam aos requisitos da pesquisa.

A avaliação do estado nutricional foi determinada por meio do índice de massa corporal (IMC). O IMC foi calculado pela divisão do peso $(P)$ pela estatura (E) ao quadrado: $P(\mathrm{~kg}) / \mathrm{E}(\mathrm{m})^{2}$. O estado nutricional dos idosos foi estabelecido de acordo com os pontos de corte recomendados pela Organização Pan-Americana de Saúde (OPAS, 2001): baixo peso (IMC $<23 \mathrm{~kg} / \mathrm{m}^{2}$ ), peso adequado (IMC 23 a 28 $\mathrm{kg} / \mathrm{m}^{2}$ ), excesso de peso $\left(I M C>28\right.$ a $30 \mathrm{~kg} / \mathrm{m}^{2}$ ) e obesidade (IMC>30 $\left.\mathrm{kg} / \mathrm{m}^{2}\right)$.

Para a avaliação do risco cardiovascular foi utilizada a medida da circunferência da cintura (CC). O risco foi determinado de acordo com os valores propostos pela WHO (2000), que considera sem risco cardiovascular os indivíduos que apresentam valores de $\mathrm{CC}<80 \mathrm{~cm}$ para mulheres e $<94 \mathrm{~cm}$ para homens; com risco aumentado os indivíduos que apresentam valores de $\mathrm{CC} \geq 80 \mathrm{~cm}$ para mulheres e $\geq 94 \mathrm{~cm}$ para homens.

Os dados foram analisados por meio da estatística descritiva e analítica. Foi utilizado o Programa Estatístico EPI Info versão 7.2.1.0. Os dados foram apresentados em tabelas de frequência absoluta (n) e relativa (\%). Para se determinar a associação entre estado nutricional e risco cardiovascular os dados foram submetidos ao teste $\chi^{2}$. Para verificar o efeito das diferentes variáveis entre dois grupos de médias foi utilizado o teste $t$ student. O nível de significância foi determinado em $5 \%(p<0,05)$.

\section{RESULTADOS E DISCUSSÃO}

Foram analisados 34 prontuários, destes $12(35,29 \%)$ eram de idosos do sexo masculino e $22(64,71 \%)$ do sexo feminino. A média de idade foi de 66,35 $\pm 5,09$ anos para ambos os sexos, sendo que a média da idade dos homens foi $67,83 \pm$ 6,07 anos e das mulheres de $65,54 \pm 4,41$ anos. Não houve significância entre as médias de idade para ambos os sexos $(p=0,2157)$.

Os indivíduos do sexo feminino foram os que mais procuraram atendimento, totalizando 22 consultas nos anos de 2014 e 2015, seguidos por 12 consultas do sexo masculino. O grupo etário que mais buscou atendimento nutricional foram os indivíduos que se encontravam na faixa dos 60 até os 69 anos de idade, sendo que $19(86,36 \%)$ correspondiam as mulheres e $9(75 \%)$ aos homens.

Resultado similar foi encontrado no estudo realizado com 300 idosos integrantes de um grupo de terceira idade no município de Estrela, no Vale do Taquari, sendo que 67,5\% dos participantes eram do gênero feminino (FREITAS et al., 2017). Soar (2015) no estudo com idosos frequentadores do Instituto Paulista de Geriatria e Gerontologia (IPGG) localizado na cidade de São Paulo, São Paulo também verificou maior frequência de mulheres (72,46\%). Palma et al. (2016) ao 
estudarem 424 idosos da cidade de Palmeira das Missões, Rio Grande do Sul, verificaram que $68,4 \%$ dos participantes eram do sexo feminino.

Da mesma forma, na Pesquisa Nacional de Saúde (PNS) realizada pelo IBGE, em parceria com o Ministério da Saúde, o percentual de mulheres (78\%) que procuram atendimento médico foi superior aos homens $(63,9 \%)$ e, a maioria $(83 \%)$ dos idosos que procuraram atendimento médico foram os que se encontravam no grupo etário dos 60 anos ou mais (IBGE, 2013b).

$\mathrm{Na}$ tabela 1, estão apresentadas as médias, desvio padrão, valores de máximo e mínimo da idade e valores antropométricos dos pacientes atendidos na Clínica de Nutrição nos anos de 2014 e 2015. Pode-se observar que a média de peso dos homens é maior $82,38 \mathrm{~kg}( \pm 17,87 \mathrm{~kg})$ em comparação com as mulheres $(74,93 \mathrm{~kg})$. Apesar disso, foram as mulheres que apresentaram a média de IMC mais elevada $31,14\left( \pm 5,97 \mathrm{~kg} / \mathrm{m}^{2}\right)$. Em relação ao risco cardiovascular, verificou-se que ambos os sexos possuem risco cardiovascular aumentado, apresentando CC $99,91 \mathrm{~cm}( \pm 15,44 \mathrm{~cm})$ para as mulheres e $102,88 \mathrm{~cm}( \pm 14,32 \mathrm{~cm})$ para homens.

TABELA 1- Médias, desvio-padrão, valores de máximo e mínimo da idade e dados antropométricos dos pacientes atendidos na clínica de nutrição nos anos de 2014 e 2015.

\begin{tabular}{|c|c|c|c|c|c|c|c|c|c|c|c|c|}
\hline \multirow[t]{2}{*}{$\begin{array}{l}\text { Idade e dados } \\
\text { antropométricos }\end{array}$} & \multicolumn{4}{|c|}{$\begin{array}{c}\text { Homens } \\
\mathrm{N}=12\end{array}$} & \multicolumn{4}{|c|}{$\begin{array}{c}\text { Mulheres } \\
\mathrm{N}=22\end{array}$} & \multicolumn{4}{|c|}{$\begin{array}{c}\text { Ambos } \\
\mathrm{N}=34\end{array}$} \\
\hline & $\bar{X}$ & $\mathrm{DP}$ & MIN & MAX & $\bar{x}$ & $\mathrm{DP}$ & MIN & MAX & $\bar{X}$ & $\mathrm{DP}$ & MIN & MAX \\
\hline Idade (anos) & 67,83 & 6,07 & 60,00 & 82,00 & 65,55 & 4,42 & 60,00 & 77,00 & 66,35 & 5,09 & 60,00 & 82,00 \\
\hline Peso (kg) & 82,38 & 17,87 & 47,80 & 114,70 & 74,93 & 13,48 & 44,00 & 98,50 & 77,56 & 15,34 & 44,00 & 114,70 \\
\hline Estatura (m) & 1,69 & 0,07 & 1,59 & 1,82 & 1,55 & 0,06 & 1,46 & 1,67 & 1,60 & 0,09 & 1,46 & 1,82 \\
\hline IMC $\left(\mathrm{kg} / \mathrm{m}^{2}\right)$ & 28,81 & 5,18 & 18,91 & 38,77 & 31,14 & 5,97 & 17,85 & 40,86 & 30,32 & 5,74 & 17,85 & 40,86 \\
\hline $\mathrm{CC}(\mathrm{cm})$ & 102,88 & 14,33 & 72,50 & 127,00 & 99,91 & 15,45 & 65,50 & 125,00 & 100,96 & 14,91 & 65,50 & 127,00 \\
\hline
\end{tabular}

$\overline{\bar{X}}$ = Média; DP = Desvio Padrão; Min = Mínimo; Max = Máximo; IMC = Índice de Massa Corpórea; CC (cm)= Circunferência da Cintura;

Em relação ao estado nutricional (tabela 2), verificou-se que o percentual $(63,63 \%)$ de mulheres que apresentaram obesidade foi maior em comparação com os homens. Apesar disso, não foi verificada associação estatisticamente significativa $(p=0,3656)$ entre estado nutricional e sexo.

O grupo etário 60 a 69 anos também foi o que mais apresentou obesidade, correspondendo a $55,89 \%$ dos idosos $(p=0,365)$. Resultado semelhante foi obtido no estudo realizado em Candelária, Rio Grande do Sul, tendo em vista que, 54,4\% da amostra de idosas participantes da pesquisa apresentavam obesidade (SANTOS et al., 2016). Em Guarapuava, Paraná, esse resultado também foi similar, no estudo realizado com 45 idosos, de ambos os sexos, participantes de um programa gratuito de educação física, em que a obesidade correspondeu a $51,1 \%$, sendo prevalecente nas mulheres (CHRISTMANN et al., 2013).

A prevalência $(41,5 \%)$ de obesidade também foi observada em um estudo realizado com idosos frequentadores de grupo de convivência de terceira idade na região do Vale dos Sinos, Rio Grande do Sul, sendo a maioria dos frequentadores do sexo feminino (FLORES et al., 2016). Da mesma forma, a obesidade foi prevalente em 43,6\% dos idosos participantes de um grupo de terceira idade de um município do interior do Rio Grande do Sul, sendo composta pela maioria do sexo feminino (ADAMl et al., 2015). 
TABELA 2- Distribuição dos idosos, segundo estado nutricional (EN), gênero e grupo etário.

\begin{tabular}{|c|c|c|c|c|c|c|c|}
\hline \multirow[t]{2}{*}{ EN/ Gênero } & \multicolumn{2}{|c|}{$\begin{array}{c}\text { Homens } \\
\mathrm{N}=12\end{array}$} & \multicolumn{2}{|c|}{$\begin{array}{c}\text { Mulheres } \\
\mathrm{N}=22\end{array}$} & \multicolumn{2}{|c|}{$\begin{array}{c}\text { Ambos } \\
\mathrm{N}=34\end{array}$} & \multirow[t]{2}{*}{$p^{*}$} \\
\hline & FA & FR & FA & FR & FA & FR & \\
\hline Baixo Peso & \multicolumn{2}{|r|}{8,33} & 2 & 9,09 & 3 & 8,82 & \multirow{5}{*}{0,356} \\
\hline Peso Adequado & 4 & 33,33 & 5 & 22,73 & 9 & 26,47 & \\
\hline Excesso de Peso & 2 & 16,67 & 1 & 4,55 & 3 & 8,82 & \\
\hline Obesidade & 5 & 41,67 & 14 & 63,63 & 19 & 55,89 & \\
\hline \multirow[t]{2}{*}{ Total } & 12 & 100,00 & 22 & 100,00 & 34 & 100,00 & \\
\hline & \multicolumn{2}{|c|}{$\begin{array}{l}60 \text { à } 69 \text { anos } \\
N=28\end{array}$} & \multicolumn{2}{|c|}{$\begin{array}{c}\geq 70 \text { anos } \\
N=6\end{array}$} & \multicolumn{2}{|c|}{$\begin{array}{c}\text { Ambos os sexos } \\
\mathrm{N}=34\end{array}$} & \multirow[t]{2}{*}{$p^{*}$} \\
\hline EN/ Grupo Etário & \multicolumn{2}{|c|}{$F R$} & FA & FR & FA & FR & \\
\hline Baixo Peso & 1 & 3,57 & 2 & 33,33 & 3 & 8,82 & \multirow{5}{*}{0,365} \\
\hline Peso Adequado & 8 & 28,57 & 1 & 16,67 & 9 & 26,47 & \\
\hline Excesso de Peso & 3 & 10,71 & - & & 3 & 8,82 & \\
\hline Obesidade & 16 & 57,15 & 3 & 50,00 & 19 & 55,89 & \\
\hline Total & 28 & 100,00 & 6 & 100,00 & 34 & 100,00 & \\
\hline
\end{tabular}

$\mathrm{N}=$ número; $\mathrm{FA}=$ frequência absoluta; $\mathrm{FR}=$ frequência relativa; ${ }^{*} T$ este $\chi^{2}$

$\mathrm{Na}$ tabela 3 , pode-se observar a distribuição dos idosos, segundo risco cardiovascular. A maioria (83,33\%) dos homens apresentou risco cardiovascular aumentado ( $C C \geq 94 \mathrm{~cm}$ ), ou seja, dos 12 atendimentos realizados, 10 estavam nessa faixa de classificação. Da mesma forma, verificou-se percentual elevado de mulheres com risco aumentado (81,82\%). Não foi observada associação estatisticamente significativa entre risco cardiovascular e sexo $(p=0,913)$. Estratificando o risco cardiovascular, segundo o grupo etário (tabela 3), verificou-se que o grupo etário dos 60 a 69 anos apresentou $24(85,71 \%)$ dos idosos com risco cardiovascular aumentado $(p=0,273)$.

TABELA 3- Distribuição dos idosos, segundo risco cardiovascular (RCV), gênero e grupo etário.

\begin{tabular}{|c|c|c|c|c|c|c|c|}
\hline \multirow[t]{2}{*}{ RCV / Gênero } & \multicolumn{2}{|c|}{$\begin{array}{c}\text { Homens } \\
\mathrm{N}=12\end{array}$} & \multicolumn{2}{|c|}{$\begin{array}{c}\text { Mulheres } \\
\mathrm{N}=22\end{array}$} & \multicolumn{2}{|c|}{$\begin{array}{c}\text { Ambos } \\
\mathrm{N}=34\end{array}$} & \multirow[t]{2}{*}{$p^{*}$} \\
\hline & FA & FR & FA & FR & FA & FR & \\
\hline Sem Risco & \multicolumn{2}{|r|}{16,67} & \multicolumn{2}{|r|}{18,18} & 6 & 17,65 & \multirow{3}{*}{0,913} \\
\hline Risco Aumentado & & 83,33 & & 81,82 & 28 & 82,35 & \\
\hline Total & & 100,00 & & 100,00 & 34 & 100,00 & \\
\hline \multirow[t]{2}{*}{ RCV / Grupo Etário } & \multicolumn{2}{|c|}{$\begin{array}{c}60 \text { à } 69 \text { anos } \\
N=28\end{array}$} & \multicolumn{2}{|c|}{$\begin{array}{c}\geq 70 \text { anos } \\
\mathrm{N}=6\end{array}$} & \multicolumn{2}{|c|}{$\begin{array}{l}\text { Ambos } \\
\mathrm{N}=34\end{array}$} & \multirow[t]{2}{*}{$p^{*}$} \\
\hline & $\mathrm{FA}$ & $\mathrm{FR}$ & $\mathrm{FA}$ & $\mathrm{FR}$ & $\mathrm{FA}$ & $\mathrm{FR}$ & \\
\hline Sem Risco & \multicolumn{2}{|r|}{33,33} & \multicolumn{2}{|r|}{9,09} & 6 & 17,65 & \multirow{3}{*}{0,273} \\
\hline Risco Aumentado & & 200,00 & & 18,18 & 28 & 82,35 & \\
\hline Total & & 233,33 & & 27,27 & 34 & 100,00 & \\
\hline
\end{tabular}

$\mathrm{N}=$ número; $\mathrm{FA}=$ frequência absoluta; $\mathrm{FR}=$ frequência relativa; ${ }^{*}$ Teste $\chi^{2}$

Associação estatisticamente significativa entre estado nutricional $(E N)$ e risco cardiovascular (RCV) foi verificada no sexo feminino $(p=0,0008)$, tendo em vista que $77,78 \%$ das mulheres apresentaram obesidade e RCV aumentado. Entre os homens 
não foi verificada essa associação $(p=0,08)$. No estudo realizado com 50 idosos, de ambos os sexos, pacientes regulares de um hospital de referência para idosos em Montes Claros, Minas Gerais, os autores verificaram que $58 \%$ das idosas apresentaram valores elevados de CC ( $\geq 80 \mathrm{~cm})$, sendo que destas $37 \%$ estavam com sobrepeso (SOARES et al., 2016).

TABELA 4- Distribuição dos idosos, segundo estado nutricional (EN), risco cardiovascular (RCV), e gênero.

\begin{tabular}{|c|c|c|c|c|c|c|c|c|c|c|c|c|}
\hline \multirow{3}{*}{ EN/RCV } & \multicolumn{4}{|c|}{$\begin{array}{c}\text { Homens } \\
\mathrm{N}=12\end{array}$} & \multicolumn{4}{|c|}{$\begin{array}{l}\text { Mulheres } \\
\qquad \mathrm{N}=22\end{array}$} & \multicolumn{4}{|c|}{$\begin{array}{c}\text { Ambos } \\
\mathrm{N}=34\end{array}$} \\
\hline & \multicolumn{2}{|c|}{ Sem Risco } & \multicolumn{2}{|c|}{ Risco Aumentado } & \multicolumn{2}{|c|}{ Sem Risco } & \multicolumn{2}{|c|}{ Risco Aumentado } & \multicolumn{2}{|c|}{ Sem Risco } & \multicolumn{2}{|c|}{$\begin{array}{c}\text { Risco } \\
\text { Aumentado }\end{array}$} \\
\hline & $\mathrm{FA}$ & FR & FA & FR & FA & FR & FA & FR & $\mathrm{FA}$ & FR & FA & FR \\
\hline & \multicolumn{4}{|c|}{$p=0,0858$} & \multicolumn{4}{|c|}{$p=0,0008$} & & & & \\
\hline Baixo Peso & 1,00 & 50,00 & - & - & 2,00 & 50,00 & - & - & 3,00 & 50,00 & - & - \\
\hline Peso Adequado & 1,00 & 50,00 & 3,00 & 30,00 & 1,00 & 25,00 & 4,00 & 22,22 & 2,00 & 33,33 & 7,00 & 25,00 \\
\hline Excesso de Peso & - & - & 2,00 & 20,00 & 1,00 & 25,00 & - & - & 1,00 & 16,67 & 2,00 & 7,14 \\
\hline Obesidade & - & - & 5,00 & 50,00 & - & - & 14,00 & 77,78 & - & - & 19,00 & 67,86 \\
\hline Total & 2,00 & 100,00 & 10,00 & 100,00 & 4,00 & 100,00 & 18,00 & 100,00 & 6,00 & 100,00 & 28,00 & 100,00 \\
\hline
\end{tabular}

$\mathrm{FA}=$ frequência absoluta; $\mathrm{FR}=$ frequência relativa; ${ }^{*}$ Teste $\chi^{2}$

$\mathrm{Na}$ tabela 5 pode-se verificar a distribuição dos idosos, segundo estado nutricional (EN), risco cardiovascular (RCV) e grupo etário. O grupo etário $\geq 70$ anos foi o que apresentou o percentual mais elevado de obesidade e RCV, porém não houve relação estatisticamente significativa $(p=N / A)$. Associação estatisticamente significativa $(p=0,016)$ entre obesidade e RCV foi verificada no grupo etário dos 60 aos 69 anos de idade. No estudo realizado em Avaré, São Paulo para avaliar a qualidade da dieta da população idosa, os autores verificaram que o consumo médio de porções de frutas, hortaliças, cereais e leite e derivado ficou abaixo do recomendado e o consumo de leguminosas, carnes e ovos, açúcar e óleo ultrapassou o recomendado, sendo que em alguns casos esse consumo apresentou valores bastante elevados (MALTA et al., 2013).

Os 10 alimentos frequentemente mais consumidos segundo estudo realizado com um grupo de idosos composto por $90 \%$ do sexo feminino, na zona leste de São Paulo foram: café (infusão), arroz branco, feijão carioca, açúcar refinado, leite integral, adoçante líquido, pão francês, leite desnatado, folha verde claro (alface), margarina com sal (FREITAS et al., 2011).

$\mathrm{Na}$ Pesquisa de Orçamentos Familiares 2008-2009 (POF, 2008-2009), os resultados mostraram que no geral a alimentação do brasileiro é composta principalmente por alimentos de alto valor energético e apresenta baixo teor de nutrientes sendo compatível com uma dieta de risco para desenvolvimento de deficiências nutricionais, obesidade e doenças crônicas não transmissíveis (IBGE, 2011).

Segundo a pesquisa de percepção do estado de saúde, estilo de vida e doenças crônicas, o consumo excessivo de sal está diretamente relacionado ao aumento do risco para desenvolvimento de DCNT como a hipertensão arterial, doenças cardiovasculares e doenças renais. A pesquisa apurou que $12,5 \%$ das mulheres entrevistadas consideravam o consumo de sal alto ou muito alto. Ainda 
segundo a mesma pesquisa, do total de pessoas com idade entre 60 e 64 anos, $44,4 \%$ informaram diagnóstico de hipertensão, entre as pessoas de 65 a 74 anos, essa proporção foi de $52,7 \%$ e $55 \%$ entre as de 75 anos ou mais (IBGE 2013a).

TABELA 5- Distribuição dos idosos, segundo estado nutricional (EN), risco cardiovascular (RCV) e grupo etário.

\begin{tabular}{|c|c|c|c|c|c|c|c|c|c|c|c|c|}
\hline \multirow{3}{*}{ EN/RCV } & \multicolumn{4}{|c|}{$\begin{array}{c}60 \text { à } 69 \\
\mathrm{~N}=28\end{array}$} & \multicolumn{4}{|c|}{$\geq 70$} & \multicolumn{4}{|c|}{$\begin{array}{l}\text { Total } \\
\mathrm{N}=34\end{array}$} \\
\hline & \multicolumn{2}{|c|}{ Sem Risco } & \multicolumn{2}{|c|}{$\begin{array}{c}\text { Risco } \\
\text { Aumentado }\end{array}$} & \multicolumn{2}{|c|}{ Sem Risco } & \multicolumn{2}{|c|}{$\begin{array}{c}\text { Risco } \\
\text { Aumentado } \\
\end{array}$} & \multicolumn{2}{|c|}{ Sem Risco } & \multicolumn{2}{|c|}{$\begin{array}{c}\text { Risco } \\
\text { Aumentado }\end{array}$} \\
\hline & FA & FR & $\mathrm{FA}$ & $\mathrm{FR}$ & $\mathrm{FA}$ & FR & FA & $\mathrm{FR}$ & FA & $\mathrm{FR}$ & FA & FR \\
\hline & \multicolumn{4}{|c|}{$p=0,016$} & \multicolumn{4}{|c|}{$p=N / A$} & & & & \\
\hline Baixo Peso & 1,00 & 25,00 & - & . & 2,00 & 100,00 & - & & 3,00 & 50,00 & - & . \\
\hline Peso Adequado & 2,00 & 50,00 & 6,00 & 25,00 & - & - & 1,00 & 25,00 & 2,00 & 33,33 & 7,00 & 25,00 \\
\hline Excesso de Peso & 1,00 & 25,00 & 2,00 & 8,33 & - & - & - & - & 1,00 & 16,67 & 2,00 & 7,14 \\
\hline Obesidade & - & & 16,00 & 66,67 & - & - & 3,00 & 75,00 & - & - & 19,00 & 67,86 \\
\hline Total & 4,00 & 100,00 & 24,00 & 100,00 & 2,00 & 100,00 & 4,00 & 100,00 & 6,00 & 100,00 & 28,00 & 100,00 \\
\hline
\end{tabular}

$\mathrm{N}=$ número; $\mathrm{FA}=$ frequência absoluta; $\mathrm{FR}=$ frequência relativa; $\mathrm{N} / \mathrm{A}=\mathrm{Não}$ Aplicável; Teste $\chi^{2}$

\section{CONCLUSÃO}

Encontrou-se percentual elevado de distúrbios nutricionais (excesso de peso e obesidade) e risco cardiovascular. Esses resultados refletem a importância da melhora na qualidade de vida desses indivíduos. Porém, outros parâmetros precisam ser avaliados para que se possa determinar com exatidão a saúde desses idosos.

\section{AGRADECIMENTOS}

Programa institucional de bolsas de iniciação científica PROBIC/UNICESUMAR.

\section{REFERÊNCIAS}

ADAMI, F. S.; FEIL, C. C.; BOSCO, S. M. Estado nutricional relacionado à qualidade de vida em idosos. Revista Brasileira de Ciências do Envelhecimento Humano, v. 12 , n. $1, \quad$ p. 28-40, 2015. Disponível em: < http://dx.doi.org/10.5335/rbceh.v12i1.4959>. doi: 10.5335/rbceh.v12i1.4959

CHRISTMANN, A. C.; ZANELATTO, C.; SEMCHECHEM, C. C.; NOVELLO, D.; SCHIESSEL, D. L. Perfil de risco de doenças cardiovasculares e estado nutricional de idosos ativos de Guarapuava-Paraná. Unopar- Científica Ciências Biológicas e da Saúde, p. 349-356, 2013. Disponível em: http://www.pgsskroton.com.br/seer/index.php/JHealthSci/article/view/570/538

FLORES, F. R.; WIECK, F. P.; PICCOLI, J. C. J.; WEBER, M. H. Qualidade da dieta entre idosos com arranjo familiar diferenciado na região do Vale do Sinos, Rio Grande do Sul. Revista Brasileira de Ciências do Envelhecimento Humano, v. 13, n. 2, p.124-134, 2016. Disponível em: <http://dx.doi.org/10.5335/rbceh.v13i2.5687>. doi: 10.5335/rbceh.v13i2.5687 
FREITAS, A. P.; VOGEL, P.; FASSINA, P.; ADAMI, F. S. Relação da Qualidade de vida com o estado nutricional de idosos. Revista Brasileira de Qualidade de Vida, v. $9, \quad$ n. 1, p.30-44, $2017 . \quad$ Disponível em: https://periodicos.utfpr.edu.br/rbqv/article/view/5236/3583

FREITAS, A. M P.; PHILIPPI, S. T.; RIBEIRO, S. M. L. Listas de alimentos relacionadas ao consumo alimentar de um grupo de idosos: análises de perspectivas. Revista Brasileira de Epidemiologia, p.161-177, 2011. Disponível em: <http://bvsms.saude.gov.br/bvs/is_digital/is_0211/pdfs/IS31(2)049.pdf>

GARRITANO, C. R.;. Análise da tendência da mortalidade por acidente vascular cerebral no Brasil no século XXI. Arquivos Brasileiros de Cardiologia, v. 98, n. 6, p.519-527, 2012. Disponível em: <http://dx.doi.org/10.1590/s0066782x2012005000041 >. doi: 10.1590/s0066-782x2012005000041

IBGE- Instituto Brasileiro de geografia e estatística. POF-Pesquisa de orçamentos familiares, 2008-2009: análise do consumo pessoal no Brasil / IBGE, Coordenação de Trabalho e Rendimento. - Rio de Janeiro: IBGE, 2011. Disponível em: < https://biblioteca.ibge.gov.br/visualizacao/livros/liv50063.pdf>.

.PNS-Pesquisa nacional de saúde, 2013a. Acesso e utilização dos serviços de saúde, acidentes e violências: Brasil, grandes regiões e unidades da federação / IBGE, Coordenação de Trabalho e Rendimento. - Rio de Janeiro: IBGE, 2015 Disponível em: https://biblioteca.ibge.gov.br/visualizacao/livros/liv94074.pdf>.

.PNS-Pesquisa nacional de saúde, 2013b. Percepção do estado de saúde, estilos de vida e doença crônica. Brasil, grandes regiões e unidades da federação / IBGE, Coordenação de Trabalho e Rendimento. - Rio de Janeiro: IBGE, 2014. Disponível em: <ftp://ftp.ibge.gov.br/PNS/2013/pns2013.pdf>.

ILC-Brasil- Centro Internacional de Longevidade Brasil. Envelhecimento Ativo: um marco Político em resposta à revolução da longevidade / Centro Internacional de Longevidade Brasil. 1 ${ }^{\underline{a}}$ edição - Rio de Janeiro, Brasil, 2015. Disponível em: < http://ilcbrazil.org/portugues/wp-content/uploads/sites/4/2015/12/EnvelhecimentoAtivo-Um-Marco-Pol\%C3\%ADtico-ILC-Brasil_web.pdf>.

MALTA, M. B.; PAPINI, S. J.; CORRENTE, J. E. Avaliação da alimentação de idosos de município paulista: aplicação do Índice de Alimentação Saudável. Ciência \& Saúde Coletiva, v. 18, n. 2, p.377-384, 2013. Disponível em: < http://dx.doi.org/10.1590/s1413-81232013000200009>. doi: 10.1590/s141381232013000200009

OPAS-Organização Pan-Americana. Informe preliminar da $36^{\mathrm{a}}$ Reunión del Comité Asesor de Ivestigaciones em Salud - Encuestra Multicéntrica - Salud Bienestar y Envejecimento (SABE) em América Latina y el Caribe, 2001. Disponível em: $<$ http://www.imsersomayores.csic.es/documentos/documentos/paho-salud-01.pdf >.

ORTOLANI, F. P. B.; GOULART, R. M. M. Doenças cardiovasculares e estado nutricional no envelhecimento: produção científica sobre o tema. Revista Kairós 
Gerontologia, v. 1, n. 18, p.307-324, 2015. Disponível em: <https://revistas.pucsp.br/index.php/kairos/article/view/26063/18703>.

PALMA, S.W.; CRUZ, S.T.; DALLEPIANE, L.B.; KIRSTEN, V.R.; KIRCHNER, R.M.; BOHRER, C.T.; MEDINA, V.B. Comparação do estado nutricional de idosos utilizando dois pontos de corte do índice de massa corporal. Santa Maria, v. 42, n. 1, p.147-154, 2016. Disponível em:

<https://periodicos.ufsm.br/revistasaude/article/viewFile/15222/pdf >.

SANTOS, B. B.; COUTO, A. N.; WICHMANN, F. M. A. Estado nutricional de idosos atendidos na Atenção Primária à Saúde do município de Candelária/RS. Cinergis, v. 17, p.364-367, 2016. Disponível em: <http://dx.doi.org/10.17058/cinergis.v17i0.8155>. doi: 10.17058/cinergis.v17i0.8155

SOAR, C. Prevalência de fatores de risco cardiovascular em idosos não institucionalizados. Revista Brasileira de Geriatria e Gerontologia, v. 18, n. 2, p.385-395, 2015. Disponível em: <http://dx.doi.org/10.1590/1809-9823.2015.14072>. doi: 10.1590/1809-9823.2015.14072

SOARES, W. D.; ROCHA, P. S.; BARBOSA, J. P; SOARES, P. K. D.; FREITAS, D. A. Estado nutricional em idosos com doenças crônicas não transmissíveis. Revista Portal: Saúde e Sociedade, v. 1, n. 2, p.146-155, 2016. Disponível em: <http://www.seer.ufal.br/index.php/nuspfamed/article/view/2381>.

WHO-World Health Organization. Global Status report on noncommunicable diseases. Geneva; 2014. Disponível em: <http://www.who.int/nmh/publications/ncdstatus-report-2014>.

WHO-World Health Organization. Obesity- preventing and managing the global epidemic: report of a WHO consultation. Geneva; 2000. Disponível em: <http://www.who.int/nutrition/publications/obesity/WHO_TRS_894/en/>. 